\title{
Medial Femoral Condyle Flap Reconstruction of a Maxillary Defect with a 3D Printing Template
}

\author{
Erin M. Taylor, MD ${ }^{1, *}$ Winona W. Wu, BA ${ }^{1, *}$ Parisa Kamali, MD ${ }^{1}$ Nalton Ferraro, MD ${ }^{2}$ \\ Joseph Upton, MD ${ }^{1}$ Samuel J. Lin, MD, MBA ${ }^{1}$ Matthew L. Iorio, MD ${ }^{1}$
}

\author{
1 Division of Plastic Surgery, Beth Israel Deaconess Medical Center, \\ Harvard Medical School, Boston, Massachusetts \\ 2 Department of Oral and Maxillofacial Surgery, Beth Israel Deaconess \\ Medical Center, Harvard Medical School, Boston, Massachusetts
}

\author{
Address for correspondence Matthew L. Iorio, MD, Division of Plastic \\ Surgery, Beth Israel Deaconess Medical Center, Harvard Medical \\ School, 330 Brookline Avenue, Stoneman 10, Boston, MA 02215 \\ (e-mail: Matt.iorio@bidmc.harvard.edu).
}

J Reconstr Microsurg Open 2017;2:e63-e68.

\begin{abstract}
Keywords

- 3D printing

- medial femoral condyle flap

- maxillary reconstruction

Background The medial femoral condyle (MFC) flap is a well-vascularized corticocancellous flap based off the descending genicular artery that can be used to reconstruct difficult bony nonunions. Although frequently used in reconstruction of extremity defects, the MFC flap is rarely used in maxillofacial reconstruction.

Methods We present a case example of an MFC vascularized corticocancellous free flap for reconstruction of an ameloblastoma maxillary defect. Additionally, we describe our use of a three-dimensional (3D) template to create an intraoperative osteotomy model for contouring the MFC flap.

Results Preoperative 3D printing of the maxillary defect and design of a methylmethacrylate template enabled the precise and rapid contouring of a well-vascularized bone flap. Postoperative 3D CT scan demonstrated satisfactory positioning of the MFC flap within the maxilla.

Conclusion The 3D printing technology continues to advance craniofacial reconstruction and allows for the efficient and precise creation of vascularized bone flaps. The MFC vascularized flap has the potential of becoming a workhorse flap in maxillary defect reconstruction alongside the use of 3D printing technology.
\end{abstract}

Maxillary defects provide a unique challenge to the reconstructive surgeon. These defects have significant consequences, including chronic fistulization, wound breakdown, infection, and facial contour irregularities. Many different attempts have been made to reconstruct maxillary defects, including soft tissue local or free flaps, osseointegrated implants, nonvascularized bone grafts, and vascularized bone grafts. Soft tissue flaps provide well-vascularized tissue to cover the bony defect; however, they do not reconstruct "like with like," atrophy over time, and they lack structure for dental implants. Osseointegrated implants may be custom-designed to fit particular defects, but the patient must have adequate bony architecture for implantation.

Both the authors contributed equally to the article.
Vascularized bone grafts have significant advantages over nonvascularized grafts, including improved biologic and mechanical properties. ${ }^{1-4}$ Vascularized bone flaps used in the reconstruction of the mandible and maxilla include the osteocutaneous scapular flap, ${ }^{5}$ osteocutaneous groin flap, ${ }^{6}$ free rib transfer, ${ }^{7}$ free fibular osteocutaneous flap, ${ }^{8}$ and distal femoral bone grafts. ${ }^{9}$ The medial femoral condyle (MFC) flap has the potential to provide many benefits in maxillary reconstruction. The corticoperiosteal free flap is based off branches of the descending genicular artery (DGA) that originate off the superficial femoral artery. The MFC flap can be harvested with cancellous bone, providing well-vascularized cancellous bone while still maintaining cortical strength. In comparison to the fibula flap, the MFC flap has a greater ratio of cancellous to cortical bone, while still maintaining a thick

Copyright $\odot 2017$ by Thieme Medical Publishers, Inc., 333 Seventh Avenue, New York, NY 10001, USA. Tel: +1(212) 584-4662.
License terms

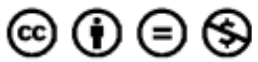

DOI https://doi.org/

March 5, 2017 accepted after revision March 30, 2017 10.1055/s-0037-1602818. ISSN 2377-0813. 
cortex (MFC: $1.6-3.0 \mathrm{~mm}$ thickness within $10 \mathrm{~cm}$ joint line for MFC, fibula: $2.3 \mathrm{~mm}$ thickness uniformly). ${ }^{10}$ Previously described MFC reconstructions include upper and lower extremity defects, ${ }^{4,11}$ scaphoid nonunions, ${ }^{12}$ recalcitrant humeral nonunions, ${ }^{13}$ foot and ankle defects, ${ }^{14}$ and thumb defects, ${ }^{15}$ in addition to maxillary and mandibular defects. ${ }^{9,16}$

Three-dimensional (3D) printing has emerged as a revolutionary technology that possesses the capacity to fabricate accurate physical models of human anatomy from medical imaging and modeling data. ${ }^{17}$ This printing has been used in plastic surgery for surgical planning, development of custom prosthetics, tissue engineering, and resident education. ${ }^{18,19}$ Craniofacial reconstruction has been a leader in 3D printing technologies in the medical field, ${ }^{20}$ where surgeons can perform complex functional and aesthetic reconstructions with high degrees of efficiency and quality. ${ }^{21,22}$

The use of patient-specific 3D models to plan maxillary reconstructions is still a novel technology that has not been heavily documented within the literature. These maxillary reconstructions included use of 3D stereolithography models for fabrication of titanium maxillary prosthesis or 3D resin models for free fibula or iliac bone crest flap reconstruction (-Table 1). ${ }^{23-27}$ We present a case of the MFC vascularized corticocancellous free flap for reconstruction of an ameloblastoma defect of the maxilla with use of a 3D printing template.

\section{Case Report}

A 17-year-old nonsmoking adolescent boy had undergone resection of ameloblastoma of the left maxilla 3 years prior, after which he remained cancer free but continued to have a considerable bony defect. This deficiency resulted in nasooral fistula and the inability to place an osseointegrated implant due to lack of bone stock.

The decision was made to use free vascularized osseous tissue from the MFC to obliterate the sinus and reconstruct the bony defect. In planning for the reconstruction, a 3D printer (Boston Children's Hospital) created a model of the craniofacial skeleton from a preoperative 3D computed tomography (CT) scan reconstruction ( - Fig. 1A). Based on the bony defect of the 3D model, a methylmethacrylate template was fashioned and sterilized for use in the operating room (-Fig. 2).

Intraoperatively, a team of surgeons prepared the recipient site whereas another team harvested the MFC flap. The donor site team identified the vastus medialis muscle and clipped branches of the descending geniculate artery (DGA) within the muscle. The DGA was found at its point of filigree on periosteum with transverse and longitudinal branches (-Fig. 3). The saphenous artery branch provided a large cutaneous perforator at this level; however, upon further evaluation of the available intraoral lining, it was decided that no cutaneous paddle was required for reconstruction. Thus, the dissection of the flap proceeded in a free osseous corticocancellous fashion without cutaneous paddle.

To maximize the use of vascularized periosteum, the methylmethacrylate template was used to design a flap based on the split point between the longitudinal and transverse potions. The MFC was osteotomized at a depth of approximately $2 \mathrm{~cm}$ with use of a sagittal saw. To prevent

Table 1 Summary of maxillary reconstruction cases using 3D printing technology reported in the literature

\begin{tabular}{|c|c|c|c|}
\hline Patient & Age/Sex & Pathology & 3D printing technology utilized \\
\hline \multirow[t]{2}{*}{ Singare et al, 2008} & $64 / M$ & $\begin{array}{l}\text { Gingival carcinoma of the right upper } \\
\text { jaw }\end{array}$ & $\begin{array}{l}\text { 3D stereolithography models of skull } \\
\text { and mirror image of left maxilla; used } \\
\text { for fabrication of titanium maxillary } \\
\text { prosthesis }\end{array}$ \\
\hline & $34 / \mathrm{F}$ & Adenocarcinoma of the maxilla & $\begin{array}{l}\text { 3D stereolithography models of skull } \\
\text { and mirror image of left maxilla; used } \\
\text { for fabrication of titanium maxillary } \\
\text { prosthesis }\end{array}$ \\
\hline He et al, 2009 & $21 / \mathrm{F}$ & $\begin{array}{l}\text { Total maxillectomy defect will absence } \\
\text { of maxilla, alveolar arch, orbital floor, } \\
\text { and } 2 / 3 \text { of the zygomatic arch and } \\
\text { palatal and nasal mucosa lining }\end{array}$ & $\begin{array}{l}\text { 3D resin models of total maxillectomy } \\
\text { defect and fibula flap }\end{array}$ \\
\hline \multirow[t]{2}{*}{ Liu et al, 2009} & $18 / \mathrm{M}$ & Osteosarcoma of the maxilla & 3D resin model of free fibula flap \\
\hline & $31 / \mathrm{M}$ & Comminuted fracture of the maxilla & 3D resin model of free fibula flap \\
\hline Jedrzejewski et al, 2012 & $72 / \mathrm{M}$ & $\begin{array}{l}\text { Squamous cell carcinoma of the left } \\
\text { maxillary sinus, penetrating to the orbit, } \\
\text { ethmoid complex, and pterygopalatine } \\
\text { fossa }\end{array}$ & $\begin{array}{l}\text { 3D models of left maxilla with tumor, } \\
\text { mirror image of right maxilla, iliac free } \\
\text { bone flap, and fibular free bone flap }\end{array}$ \\
\hline Nkenke and Eitner, 2014 & $23 / M$ & $\begin{array}{l}\text { Loss of upper right-side dentition, right } \\
\text { alveolar crest, and right facial zygomatic } \\
\text { wall through trauma }\end{array}$ & $\begin{array}{l}\text { 3D models of skull, fibula, and fabri- } \\
\text { cated vascularized fibula flap }\end{array}$ \\
\hline
\end{tabular}

Abbreviations: 3D, three-dimensional; F, female; $M$, male.

Note: Demographics, patient pathologies, and the methods by which 3D printing was used for preoperative planning enumerated for each case. Case reports were identified using the following search strategies on PubMed: 3D printing AND maxilla reconstruction, 3D model AND maxilla reconstruction. 


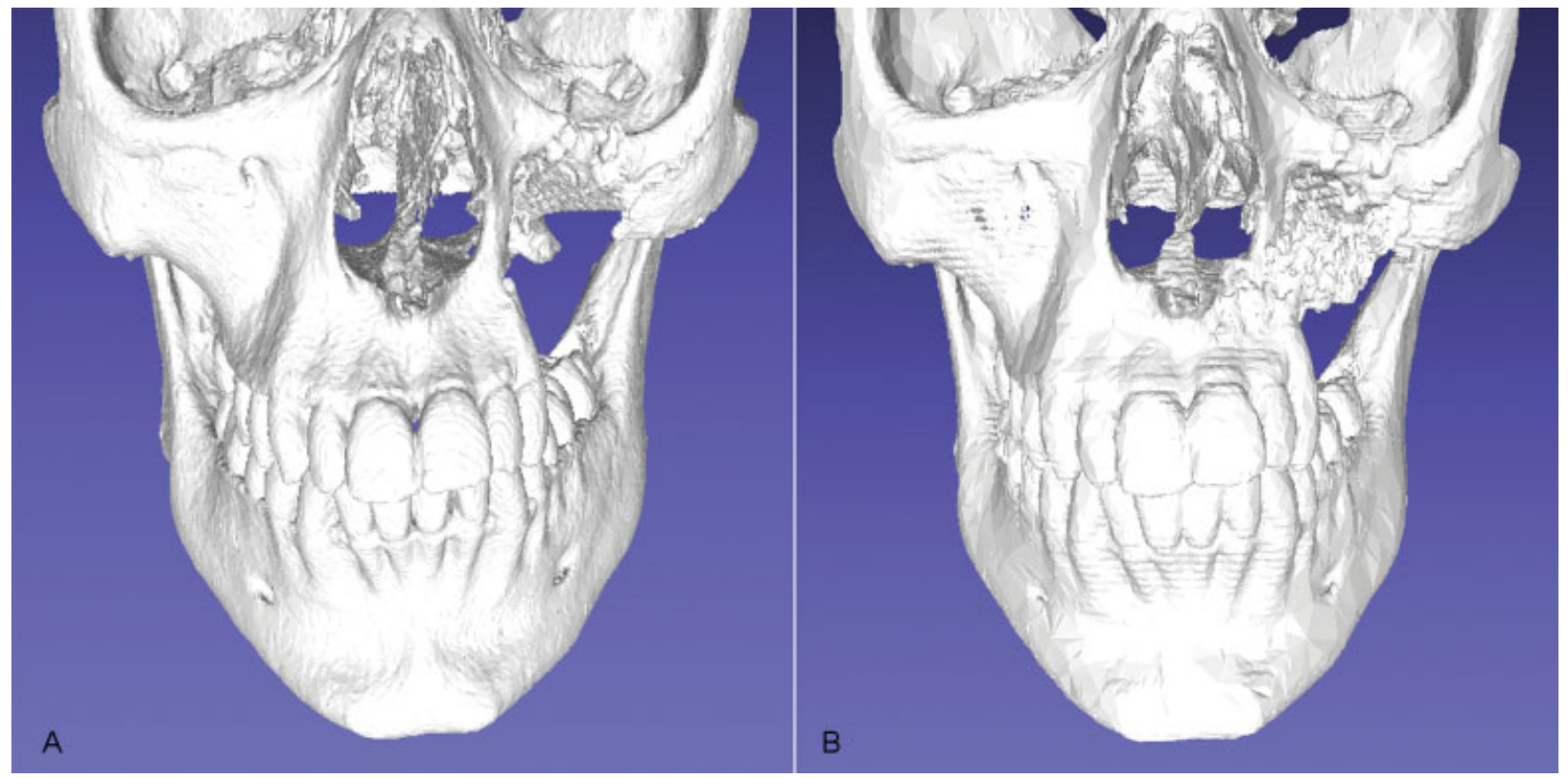

Fig. 1 (A) Pre- and (B) postoperative 3D computed tomography reconstructed scans.

delamination of the cortical and cancellous layers, the flap was carefully elevated with curved osteotomes. Rongeurs further contoured the flap to match the methylmethacrylate template while still in situ (-Fig. 4). In preparation for transfer, the descending geniculate artery was dissected to the superficial femoral artery and Hunter's canal.

Concurrently, the team preparing the recipient site raised intraoral flaps through the prior alveolar sulcus incision. The residual maxilla was entered, scar removed, and surrounding bone and tissue resected to reveal healthy tissue for recipient of a vascularized bone graft. The preoperative templates were placed and verified to match the harvested corticocancellous graft from the right femur. Through a preauricular incision, the superficial temporal artery and vein were identified and dissected as recipient vessels. The femoral vascularized graft was transected and transferred to the left maxilla for an end-to-end anastomosis between the superficial temporal vessels and the descending genicular vessels. The site of femoral harvest was packed with allogenic bone graft and closed. The intraoral flap was inset and skin closed. Doppler flow was established throughout the course of the pedicle with an external stitch placed for flap monitoring.

The postoperative 3D CT scan demonstrated satisfactory positioning of the MFC flap within the maxilla (-Fig. 1B). The patient recovered well from the free flap with minimal donor site morbidity, including immediate postoperative pain that improved. He ambulated on postoperative day 1 with a knee brace. Of note, his postoperative course was significant for breakdown of the intraoral incision with exposure of the bone graft, which required a radial forearm free flap (RFFF) for soft tissue coverage 1.5 months later. The patient recovered well after the RFFF and has had no other complications.

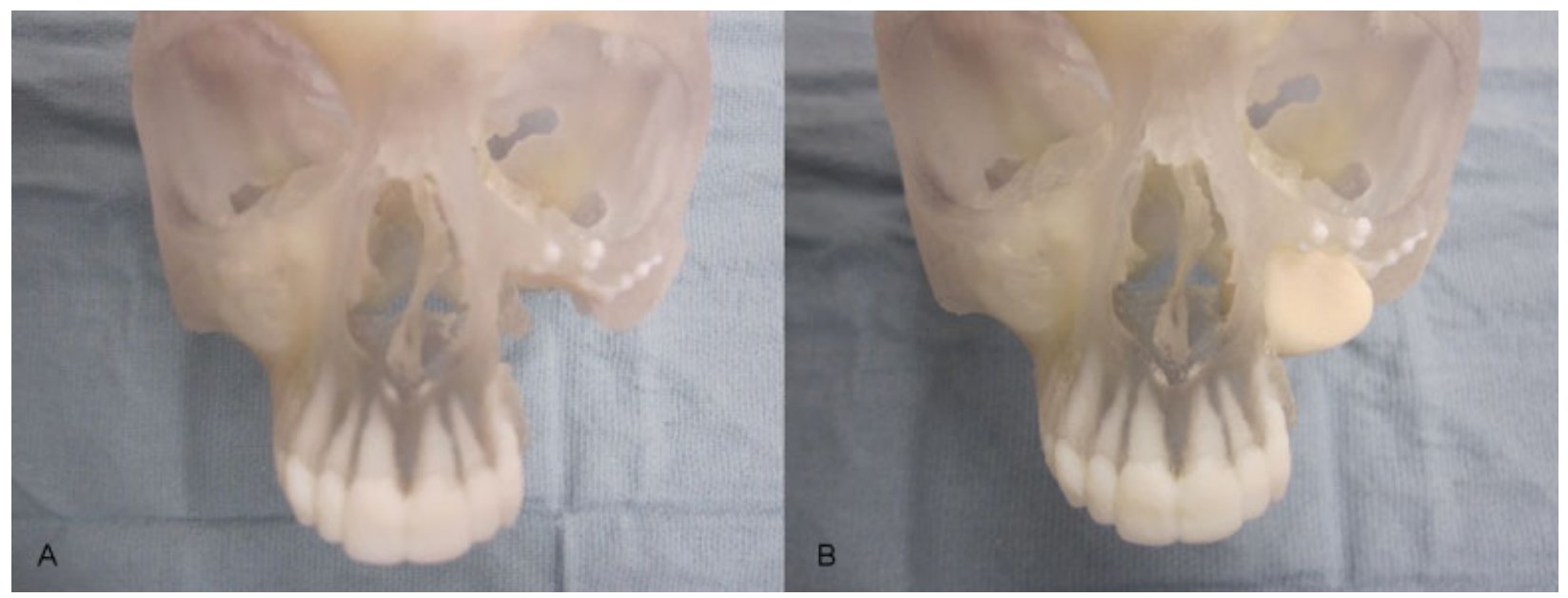

Fig. 2 Three-dimensional printer reconstruction of (A) maxillary defect and (B) maxillary defect with methylmethacrylate template. 


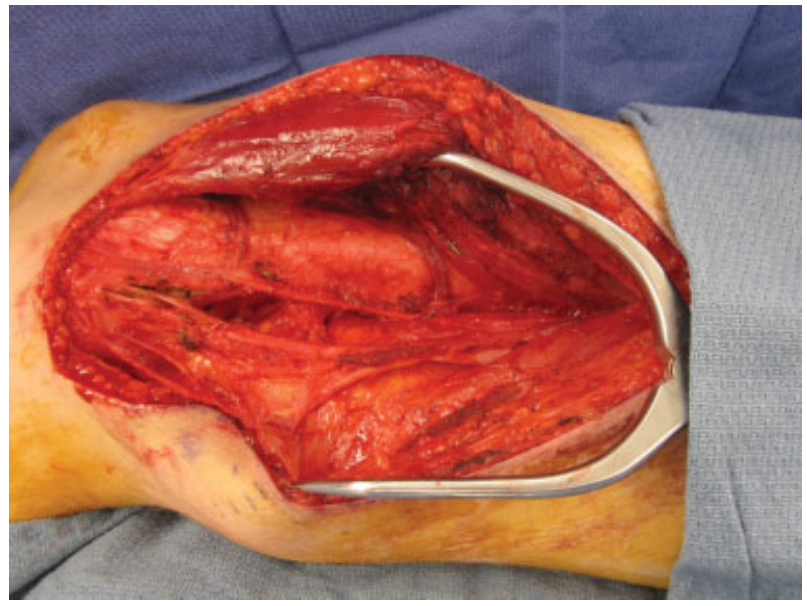

Fig. 3 Medial femoral condyle (MFC) corticocancellous vascularized flap harvest. The MFC flap is based of branches of the descending genicular artery (DGA), originating off the superficial femoral artery, which was dissected to Hunter's canal.

\section{Discussion}

The MFC flap provides well-vascularized corticocancellous bone for the reconstruction of maxillary defects. The maxilla offers a particularly challenging site for reconstruction given its anatomic location, shape, and function. In this patient, fistulization at the site of the defect required well-vascularized tissue coverage. Replacing deficient bone with a vascularized bone flap allows for restoration of maxilla architecture with similar biologic and mechanical characteristics as well as excellent bone quality for future osteointegrated implants.

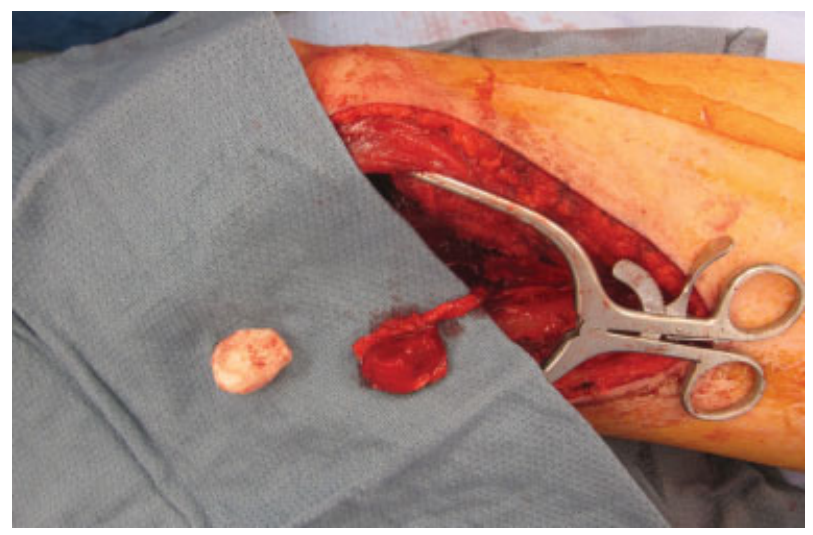

Fig. 4 Medial femoral condyle (MFC) corticocancellous vascularized flap based on branches of the descending genicular artery (DGA). To maximize the use of vascularized periosteum, the methylmethacrylate template was used to design a flap based on the split point between the longitudinal and transverse potions. The MFC was osteotomized at a depth of approximately $2 \mathrm{~cm}$ with a sagittal saw and the flap was carefully elevated with curved osteotomes. The flap was further contoured to match the methylmethacrylate template with rongeurs.
Other vascularized bone flaps, such as the scapular, free rib, iliac, and fibular flaps, do not have the same ability to reconstruct complex 3D structures. The corticocancellous vascularized tissue of the MFC flap can be contoured to fit unique bony deficits. The perfusion of the DGA has been found to be large and reliable, approximately $13.7 \mathrm{~cm}$ proximal to the joint line, allowing the MFC to provide vascularized reconstruction for larger bony deficits that may have traditionally been reserved for fibula flap reconstruction. ${ }^{28}$ Furthermore, the MFC flap results in minimal donor site morbidity, as experienced with our patient. A previous study used radiographic evidence to demonstrate $100 \%$ osseous integration, no findings of degenerative joint disease, and minimal unassisted bone regeneration at the donor site at greater than 1 year postoperatively. ${ }^{29}$

Santamaria and Cordeiro described a classification system of maxillectomy defects for midface reconstruction. ${ }^{30}$ The algorithm divides maxillary defects into four types based on the extent of maxillary bone resection in addition to associated defects of the soft tissue, palate, and orbital contents. Type I defects are "limited" maxillectomy defects, with orbital floor and palate intact, which have small volume and large surface area requirements. These defects are typically reconstructed with the radial forearm flap and less frequently with the lateral arm flap, anterolateral thigh (ALT) flap, or scapular flap. Type II defects are "subtotal" maxillectomy defects involving the palate, which have medium volume and large surface area requirements. Type IIA includes a palatal defect of less than $50 \%$, for which a skin graft and palatal obturator may be used for reconstruction. Type IIB includes a palatal defect of greater than $50 \%$, for which a radial forearm osteocutaneous flap is recommended. The free fibula flap has been described as too bulky for this reconstruction. Type III defects are "total" maxillectomy defects with involvement of the palate and orbital floor. Type IIIA defects are total maxillectomy with sparing of orbital contents, which have medium-large volume and medium-large surface defects. These defects require both orbital floor and palate reconstruction, for which nonvascularized bone graft and soft tissue reconstruction with a rectus abdominis flap are used. Type IIIB defects are total maxillectomy with orbital exenteration, which have large volume and large surface area defects. Reconstruction requires closure of the palate, nasal lining, eye defect, and anterior cranial base, for which a rectus abdominis flap is used. Type IV defects are "orbitomaxillectomy," which are large volume and large surface area defects with an intact palate. These defects require soft tissue fill for which a rectus abdominis flap is used. ${ }^{30-32}$

In our case the patient had a type IIIA Total maxillectomy defect with sparing of orbital contents, for which he had prior reconstruction of the orbital floor. The MFC flap provided wellvascularized corticocancellous bone to reconstruct the missing maxillary bone and palate, allowing for restoration of cheek volume and future dental implants. We propose that the MFC flaps can be used for a variety of maxillary defect types, as the vascularized bone graft can be used to reconstruct small to large volume defects as well as orbital floor and palatal reconstruction. 
The 3D printer was instrumental in creating an accurate preoperative craniofacial skeleton to base the methylmethacrylate template for maxillary reconstruction. For many years, reconstructions of the maxilla have largely been achieved using autologous grafts, alloplastic materials, and titanium implants that were manually shaped perioperatively at the surgical site. ${ }^{23}$ However, this conventional methodology possesses notable challenges and flaws. Perioperative adaptation of maxillary implants without preexisting knowledge or accurate visualization of a patientspecific 3D craniofacial anatomy can lead to difficulties in contouring free bone flaps to their desired shape and in filling defected areas properly. These realities may not only encumber the accuracy and efficiency of the reconstruction but may also lead to more timely and invasive procedures with increased risks of postsurgical complications. ${ }^{33}$

In this case we demonstrated that $3 \mathrm{D}$ printing reconstruction of a craniofacial defect allows for satisfactory model for effective preoperative surgical planning. The unique ability to mold the corticocancellous bone of the MFC flap allows accurate reconstruction of the defect based on the preoperative models. The 3D model of the free bony flap allowed for the timely transection of a customized graft that could fit accurately into the left maxillary region. This maxillary reconstruction reflects similar procedures that take advantage of preoperative 3D models to plan maxillary and mandibular reconstruction using other flaps, such as the free fibula, in the planning of osteotomy sites. Preoperative 3D models have the great potential to reduce intraoperative time and errors with an accurate preoperative template, with the result of superior functional and aesthetic outcomes. ${ }^{23-27}$ This process has become more convenient and cost-effective through surgeon-based 3D printing, which allows for complete surgeon control of the microvascular perioperative planning process using home 3D printing technology. ${ }^{34}$

There are still some limitations to the use of physical 3D models for the preoperative planning of maxillary reconstructions. While 3D templates can accurately replicate defective areas and the appropriate grafts that are needed, they lack of soft tissue details. Our patient had breakdown of his incision, exposing the bone graft, that required soft tissue coverage with an REFF 1.5 months later. This complication can be avoided by taking a skin paddle with the MFC flap based off the saphenous cutaneous perforator $^{35,36}$ or the more distal DGA-communicating branch. ${ }^{37}$ Our case ultimately demonstrates that a successful maxillary reconstruction can be accomplished with the guidance of 3D modeling and patient-specific 3D printed anatomical templates. The 3D technology should be used more frequently in the planning of free flap reconstruction of complex 3D osseous defects.

\section{Conclusion}

The MFC vascularized corticocancellous flap provides an excellent option for reconstruction of maxillary defects with minimal donor site morbidity. The advent of 3D printing technology allows the creation of a more precise template and thus bone flap to fit the defect, which is a perfect tool for the easily shaped and pliable MFC flap. The unique ability to mold the corticocancellous bone of the MFC flap allows accurate reconstruction of the defect based on the preoperative models. If soft tissue coverage is in question, MFC flaps can be taken with a cutaneous component based off the saphenous branch. Thus, MFC vascularized flaps have the potential of becoming a workhorse flap in maxillary defect reconstruction alongside the use of 3D printing technology.

\section{References}

1 Goldberg VM, Shaffer JW, Field G, Davy DT. Biology of vascularized bone grafts. Orthop Clin North Am 1987;18(02):197-205

2 Shaffer JW, Davy DT, Field GA, Bensusan JS, Kellis GJ. The superiority of vascularized compared to nonvascularized rib grafts in spine surgery shown by biological and physical methods. Spine 1988;13(10):1150-1154

3 Shaffer JW, Field GA, Goldberg VM, Davy DT. Fate of vascularized and nonvascularized autografts. Clin Orthop Relat Res 1985;(197):32-43

4 Choudry UH, Bakri K, Moran SL, Karacor Z, Shin AY. The vascularized medial femoral condyle periosteal bone flap for the treatment of recalcitrant bony nonunions. Ann Plast Surg 2008;60(02):174-180

5 Swartz WM, Banis JC, Newton ED, Ramasastry SS, Jones NF, Acland R. The osteocutaneous scapular flap for mandibular and maxillary reconstruction. Plast Reconstr Surg 1986;77(04):530-545

6 Taylor GI. Reconstruction of the mandible with free composite iliac bone grafts. Ann Plast Surg 1982;9(05):361-376

7 Daniel RK. Free rib transfer by microvascular anastomoses. Plast Reconstr Surg 1977;59(05):737-738

8 Bähr W. Maxillary augmentation with a microvascularly anastomosed fibula: a preliminary report. Int J Oral Maxillofac Implants 1996;11(06):719-727

9 Gaggl AJ, Bürger HK, Chiari FM. Free microvascular transfer of segmental corticocancellous femur for reconstruction of the alveolar ridge. Br J Oral Maxillofac Surg 2008;46(03):211-217

10 Masden DL, Iorio ML, Higgins JP. Comparison of the osseous characteristics of medial femoral condyle and fibula flaps. J Hand Surg Eur Vol 2013;38(04):437-439

11 Bakri K, Shin AY, Moran SL. The vascularized medial femoral corticoperiosteal flap for reconstruction of bony defects within the upper and lower extremities. Semin Plast Surg 2008;22(03):228-233

12 Jones DB Jr, Moran SL, Bishop AT, Shin AY. Free-vascularized medial femoral condyle bone transfer in the treatment of scaphoid nonunions. Plast Reconstr Surg 2010;125(04):1176-1184

13 Kakar S, Duymaz A, Steinmann S, Shin AY, Moran SL. Vascularized medial femoral condyle corticoperiosteal flaps for the treatment of recalcitrant humeral nonunions. Microsurgery 2011;31(02):85-92

14 Haddock NT, Alosh H, Easley ME, Levin LS, Wapner KL. Applications of the medial femoral condyle free flap for foot and ankle reconstruction. Foot Ankle Int 2013;34(10):1395-1402

15 Ruston JC, Amin K, Darhouse N, Sivakumar B, Floyd D. The vascularized medial femoral corticoperiosteal flap for thumb reconstruction. Plast Reconstr Surg Glob Open 2015;3(08):e492

16 Martin D, Bitonti-Grillo C, De Biscop J, et al. Mandibular reconstruction using a free vascularised osteocutaneous flap from the internal condyle of the femur. Br J Plast Surg 1991;44(06):397-402

17 Winder J, Bibb R. Medical rapid prototyping technologies: state of the art and current limitations for application in oral and maxillofacial surgery. J Oral Maxillofac Surg 2005;63(07):1006-1015

18 Gerstle TL, Ibrahim AM, Kim PS, Lee BT, Lin SJ. A plastic surgery application in evolution: three-dimensional printing. Plast Reconstr Surg 2014;133(02):446-451

19 Chae MP, Rozen WM, McMenamin PG, Findlay MW, Spychal RT, Hunter-Smith DJ. Emerging applications of bedside 3D printing in plastic surgery. Front Surg 2015;2:25. Doi: 10.3389/fsurg. 2015.00025 
20 Choi JW, Kim N. Clinical application of three-dimensional printing technology in craniofacial plastic surgery. Arch Plast Surg 2015;42(03):267-277

21 Liu YF, Xu LW, Zhu HY, Liu SS. Technical procedures for templateguided surgery for mandibular reconstruction based on digital design and manufacturing. Biomed Eng Online 2014;13:63. Doi: 10.1186/1475-925X-13-63

22 Ventola CL. Medical applications for 3D printing: current and projected uses. P\&T 2014;39(10):704-711

23 Singare S, Liu Y, Li D, Lu B, Wang J, He S. Individually prefabricated prosthesis for maxilla reconstruction. J Prosthodont 2008;17(02): 135-140

24 He Y, Zhu HG, Zhang ZY, He J, Sader R. Three-dimensional model simulation and reconstruction of composite total maxillectomy defects with fibula osteomyocutaneous flap flow-through from radial forearm flap. Oral Surg Oral Med Oral Pathol Oral Radiol Endod 2009;108(06):e6-e12

25 Liu XJ, Gui L, Mao C, Peng X, Yu GY. Applying computer techniques in maxillofacial reconstruction using a fibula flap: a messenger and an evaluation method. J Craniofac Surg 2009;20(02):372-377

26 Jędrzejewski P, Maciejewski A, Szymczyk C, Wierzgoń J. Maxillary reconstruction using a multi-element free fibula flap based on a three-dimensional polyacrylic resin model. Pol Przegl Chir 2012; 84(01):49-55

27 Nkenke E, Eitner S. Complex hemimaxillary rehabilitation with a prefabricated fibula flap and cast-based vacuum-formed surgical template. J Prosthet Dent 2014;111(06):521-524

28 Iorio ML, Masden DL, Higgins JP. The limits of medial femoral condyle corticoperiosteal flaps. J Hand Surg Am 2011;36(10): 1592-1596
29 Rao SS, Sexton CC, Higgins JP. Medial femoral condyle flap donorsite morbidity: a radiographic assessment. Plast Reconstr Surg 2013;131(03):357e-362e

30 Santamaria E, Cordeiro PG. Reconstruction of maxillectomy and midfacial defects with free tissue transfer. J Surg Oncol 2006; 94(06):522-531

31 Cordeiro PG, Chen CM. A 15-year review of midface reconstruction after total and subtotal maxillectomy: part II. Technical modifications to maximize aesthetic and functional outcomes. Plast Reconstr Surg 2012;129(01):139-147

32 McCarthy CM, Cordeiro PG. Microvascular reconstruction of oncologic defects of the midface. Plast Reconstr Surg 2010; 126(06):1947-1959

33 Müller A, Krishnan KG, Uhl E, Mast G. The application of rapid prototyping techniques in cranial reconstruction and preoperative planning in neurosurgery. J Craniofac Surg 2003;14(06): 899-914

34 Taylor EM, Iorio ML. Surgeon-based 3D printing for microvascular bone flaps. J Reconstr Microsurg 2017. Doi: 10.1055 s-0037-1600133

35 Sakai S, Soeda S, Matsukawa A. External oblique musculocutaneous flap for the reconstruction of a lumbo-sacral defect. $\mathrm{Br}$ J Plast Surg 1988;41(05):551-553

36 Sananpanich K, Atthakomol P, Luevitoonvechkij S, Kraisarin J. Anatomical variations of the saphenous and descending genicular artery perforators: cadaveric study and clinical implications for vascular flaps. Plast Reconstr Surg 2013;131(03):363e-372e

37 Iorio ML, Masden DL, Higgins JP. Cutaneous angiosome territory of the medial femoral condyle osteocutaneous flap. J Hand Surg Am 2012;37(05):1033-1041 